\title{
Simultaneous measurement of temperature and refractive index based on microfiber knot resonator integrated in an abrupt taper Mach- Zehnder interferometer
}

André D. Gomes, Orlando Frazão

André D. Gomes, Orlando Frazão, "Simultaneous measurement of temperature and refractive index based on microfiber knot resonator integrated in an abrupt taper Mach-Zehnder interferometer," Proc. SPIE 10453, Third International Conference on Applications of Optics and Photonics, 1045324 (22 August 2017); doi: 10.1117/12.2275813

SPIE Event: Third International Conference on Applications of Optics and Photonics, 2017, Faro, Portugal 


\title{
Simultaneous Measurement of Temperature and Refractive Index Based on Microfiber Knot Resonator Integrated in an Abrupt Taper Mach-Zehnder Interferometer
}

\author{
André D. Gomes ${ }^{* a}$, and Orlando Frazão ${ }^{\mathrm{a}}$ \\ aINESC TEC and Department of Physics and Astronomy, Faculty of Sciences, University of Porto, \\ Rua do Campo Alegre 687, 4169-007 Porto, Portugal;
}

\begin{abstract}
A microfiber knot resonator integrated in an abrupt taper-based Mach-Zehnder interferometer was used for simultaneous measurement of temperature and refractive index. This compact structure was fabricated using only $\mathrm{CO}_{2}$ laser processing. The transmission spectrum is the combination of the microfiber knot resonator and the Mach-Zehnder interferometer responses. The two different components of the transmission spectrum (the microfiber knot resonator and the MachZehnder interferometer components) present different sensitivities when subjected to physical or chemical parameters. A characterization in temperature, refractive index, and sodium chloride $(\mathrm{NaCl})$ concentration was performed. A simple matrix method was used for simultaneous measurement of temperature and refractive index.
\end{abstract}

Keywords: Optical fiber sensor, Mach-Zehnder interferometer, microfiber knot resonator, temperature sensor, refractive index sensor, simultaneous measurement.

\section{INTRODUCTION}

In the past few years microfiber knot resonators (MKR) have been widely explored as sensors. The taper in the knotted region allows the evanescent field of light to couple between the adjacent sections of the taper creating a resonance ${ }^{1}$. A wide variety of parameters were able to be measured by monitoring the sensor resonance, such as temperature ${ }^{2,3}$, concentration of sodium chloride and refractive index $(\mathrm{RI})^{4-6}$, among others. A simple MKR in a Sagnac loop reflector was reported ${ }^{4}$ for refractive index sensing with a sensitivity of $30.49 \mathrm{~nm} / \mathrm{RIU}$ in a range from 1.334-1.348. For the same purpose, a Teflon-coated MKR was proposed with a sensitivity of $30.5 \mathrm{~nm} / \mathrm{RIU}$ in a range from 1.3322 to 1.3412 . High sensitivity can be achieved by using different configurations, such as cascaded MKRs presented by Xu et al. ${ }^{6}$. Such device achieved a sensitivity of $6523 \mathrm{~nm} / \mathrm{RIU}$ in a refractive index range from 1.3320 to 1.3350 .

The Mach-Zehnder interferometer (MZI) technique is mainly used for measuring the refractive index of a medium due to the induced phase shift of the guided light created by the analyzed medium ${ }^{7}$. Nevertheless, year after year new configurations were developed increasing, therefore, the scope of this sensor. Different optical fiber MZI configurations have been reported for refractive index ${ }^{8-10}$ and temperature sensing. For refractive index, a simple biconical fiber taper ${ }^{8}$ can achieve sensitivities of around $705 \mathrm{~nm} / \mathrm{RIU}$ in a range of 1.333 to 1.350 . Higher sensitivities can be obtained by creating a taper-based MZI in a thinned optical fiber ${ }^{9}$. Such sensor can achieve a maximum sensitivity of $2210.84 \mathrm{~nm} / \mathrm{RIU}$ between 1.3997 and 1.4096. Different configurations, such as an MZI based on a large knot fiber resonator can also be used for refractive index sensing ${ }^{10}$, presenting a sensitivity of $642 \mathrm{~nm} / \mathrm{RIU}$ in a wide range of refractive indices $(1.3735$ to 1.428). Recently, several configurations using MZIs have been demonstrated for simultaneous measurement of temperature and refractive index ${ }^{11-14}$. In 2016, a microfiber MZI with a knot resonator was presented for the same purpose $^{15}$.

In this work, a distinct approach is proposed for temperature and refractive index or $\mathrm{NaCl}$ concentration discrimination using the combination of two different optical structures: a microfiber knot resonator combined with a Mach-Zehnder interferometer based on an abrupt fiber taper. This compact sensor is produced using the same technology $-\mathrm{CO}_{2}$ laser processing.

*ardcgomes@gmail.com

Third International Conference on Applications of Optics and Photonics, edited by Manuel F. M. Costa, Proc. of SPIE Vol. 10453, 1045324 - @ 2017 SPIE · CCC code: 0277-786X/17/\$18 · doi: 10.1117/12.2275813 


\section{EXPERIMENTAL SETUP AND RESULTS}

A microfiber knot resonator (MKR) with a diameter of around $680 \mu \mathrm{m}$ was fabricated using a $50 \mathrm{~mm}$-long abrupt fiber taper. An abrupt taper with a waist diameter of $8.6 \mu \mathrm{m}$ was produced using a $\mathrm{CO}_{2}$ laser system. The abrupt transition regions are $900 \mu \mathrm{m}$-long, allowing the excitation of modes with different effective refractive indices in the beginning of the taper. These modes will then recouple in the knot region due to the intrinsic curvature of the structure, creating an MZI.

Figure 1 shows a schematic of the proposed sensor and its transmission spectrum in water at $44{ }^{\circ} \mathrm{C}$. The sensor was connected between a broadband optical source and an optical spectrum analyzer (OSA). The optical source had a central wavelength of $1550 \mathrm{~nm}$ and a bandwidth of $100 \mathrm{~nm}$. Transmission measurements were realized with a resolution of $0.04 \mathrm{~nm}$. From Figure 1, it is possible to observe that the transmission spectrum is the combined response of the MZI and the MKR response. Since the MKR resonant frequency is higher than the MZI interference pattern, a fast fourier transform (FFT) lowpass filter can be used to remove the MKR resonance allowing to measure the MZI component. The MZI is caused by the effective refractive index difference between the different modes excited by the abrupt taper, mainly the fundamental and the second order mode $\left(\mathrm{LP}_{01}\right.$ and $\left.\mathrm{LP}_{11}\right)$.
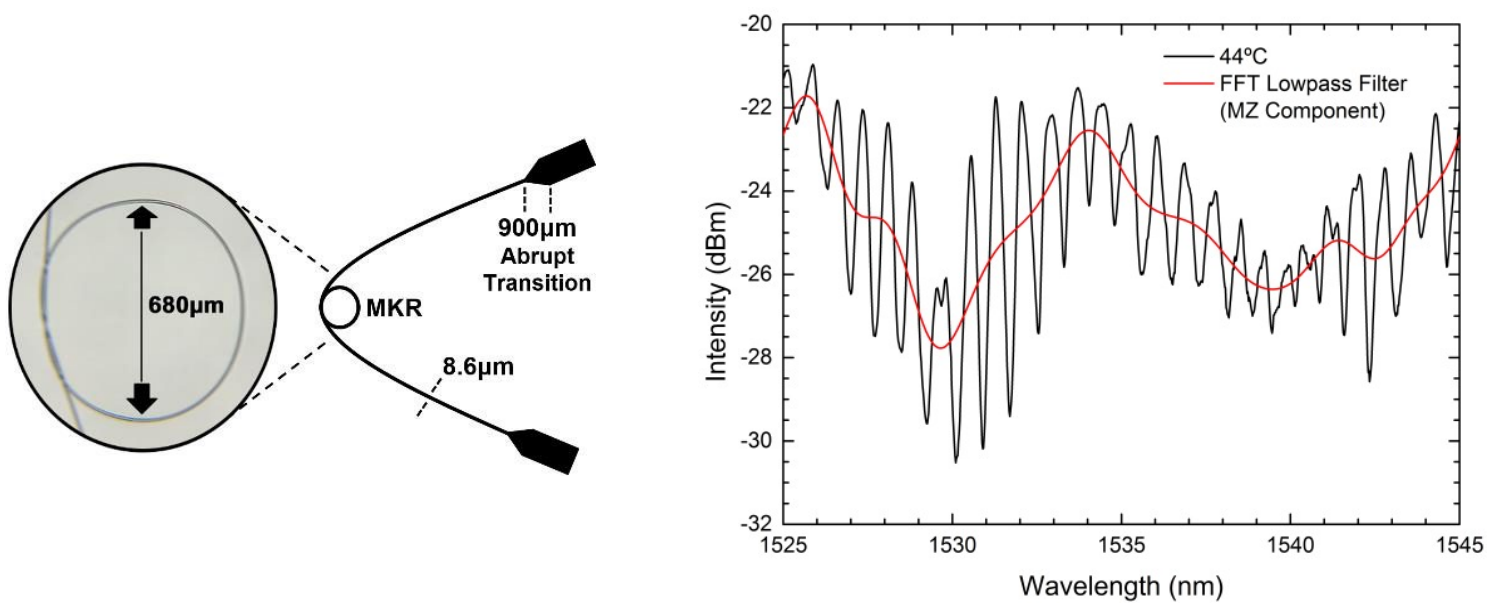

Figure 1. Schematic of the sensor and its transmission spectrum in water at $44^{\circ} \mathrm{C}$. Filtered MZI spectral component (lowpass filter cutoff frequency: $0.5 \mathrm{~nm}-1$ ).

Temperature measurements were performed by dipping the sensor in water while progressively decreasing the temperature from $50{ }^{\circ} \mathrm{C}$ to $38^{\circ} \mathrm{C}$. Figure 2(a) shows the transmission spectra at two different temperatures. The MZI peak red shifts with the decrease of temperature [fig. 2(a)] while the MKR peak blue shifts, as observed in figure 2(b).

In terms of refractive index, a temperature-RI conversion scale was used based on the RI dependence of water with temperature, constant pressure, and wavelength ${ }^{16}$. The conversion of water temperature in degrees Celsius to RI is given by the expression:

$$
n(T)=1.33315-3.14566 \times 10^{-5} T-1.35711 \times 10^{-6} T^{2}+3.626 \times 10^{-10} T^{3}
$$

From equation 1, the sensor response to refractive index variations was obtained. For refractive index, the MZI peak blue shifts with the decrease of the external refractive index while the MKR peak red shifts.

The wavelength shift as a function of the temperature variation for both components, the MZI and the MKR, is depicted in figure 3(a). For temperature sensing, temperature sensitivities of $-196 \pm 2 \mathrm{pm} /{ }^{\circ} \mathrm{C}$ and $25.1 \pm 0.9 \mathrm{pm} /{ }^{\circ} \mathrm{C}$ were obtained for the MZI and the MKR, respectively. For refractive index sensing, the wavelength shift as a function of the external refractive index variation for both components is shown in figure 3(b). Refractive index sensitivity was $1287 \pm 14 \mathrm{~nm} / \mathrm{RIU}$ and $-179 \pm 3 \mathrm{~nm} / \mathrm{RIU}$ were achieved for the MZI and the MKR, respectively, in a refractive index range from 1.32823 to 1.33001 .

A possible sensing application for the structure is in seawater research, monitoring the amount of $\mathrm{NaCl}$ dissolved in water. A $\mathrm{NaCl}(\mathrm{wt} \%) \mathrm{RI}$ conversion table ${ }^{17}$ was used to analyze the sensor in terms of $\mathrm{NaCl}$ concentration. The concentration of $\mathrm{NaCl}$ per mil (\%) depends linearly on the refractive index with a gradient of $0.000177 \mathrm{RIU} / \mathrm{NaCl}(\%)$. Figure $3(\mathrm{~b})$ also presents the wavelength shift as a function of the $\mathrm{NaCl}$ concentration in water. The sensitivity to $\mathrm{NaCl}$ concentration was 
$227 \pm 2 \mathrm{pm} / \mathrm{NaCl} \%$ and $-31.6 \pm 7 \mathrm{pm} / \mathrm{NaCl} \%$ for the MZI and the MKR components respectively, for $\mathrm{NaCl}$ concentrations from 0 to $10.08 \%$.
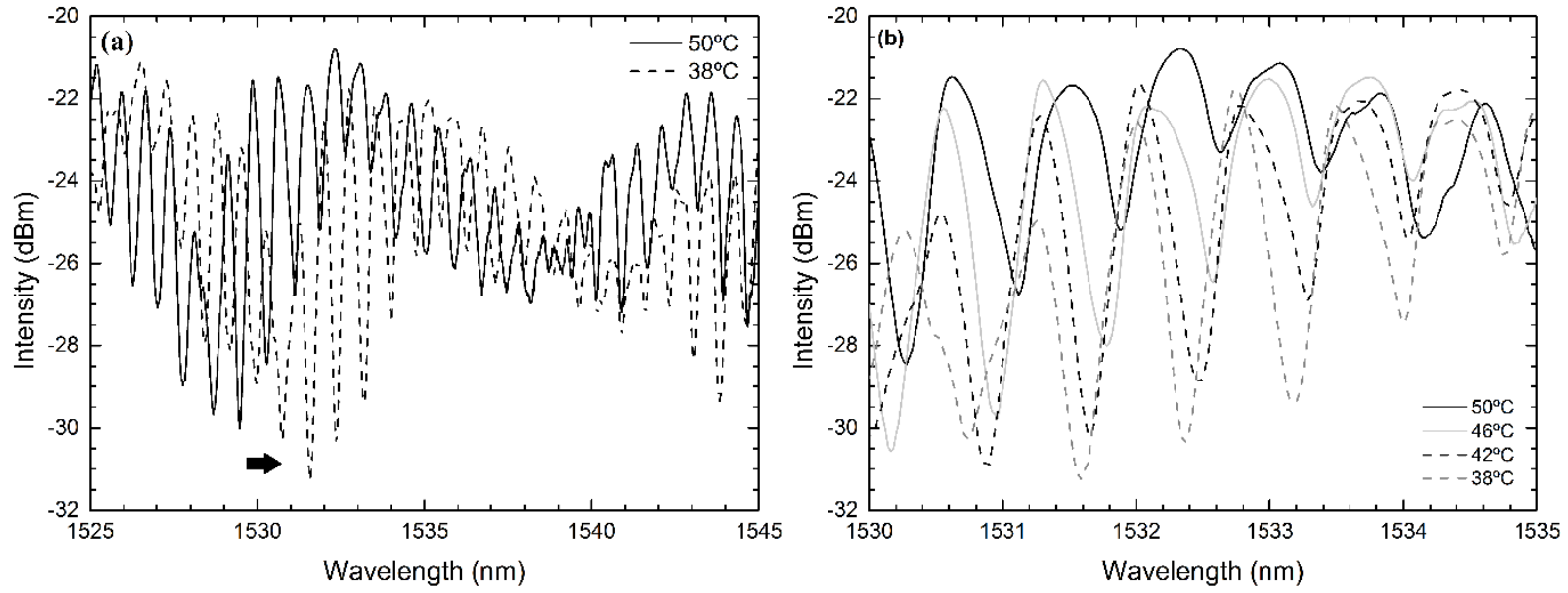

Figure 2. Transmission spectra in water at different temperatures: (a) Full spectra (MZI and MKR). (b) Microfiber knot resonator component.
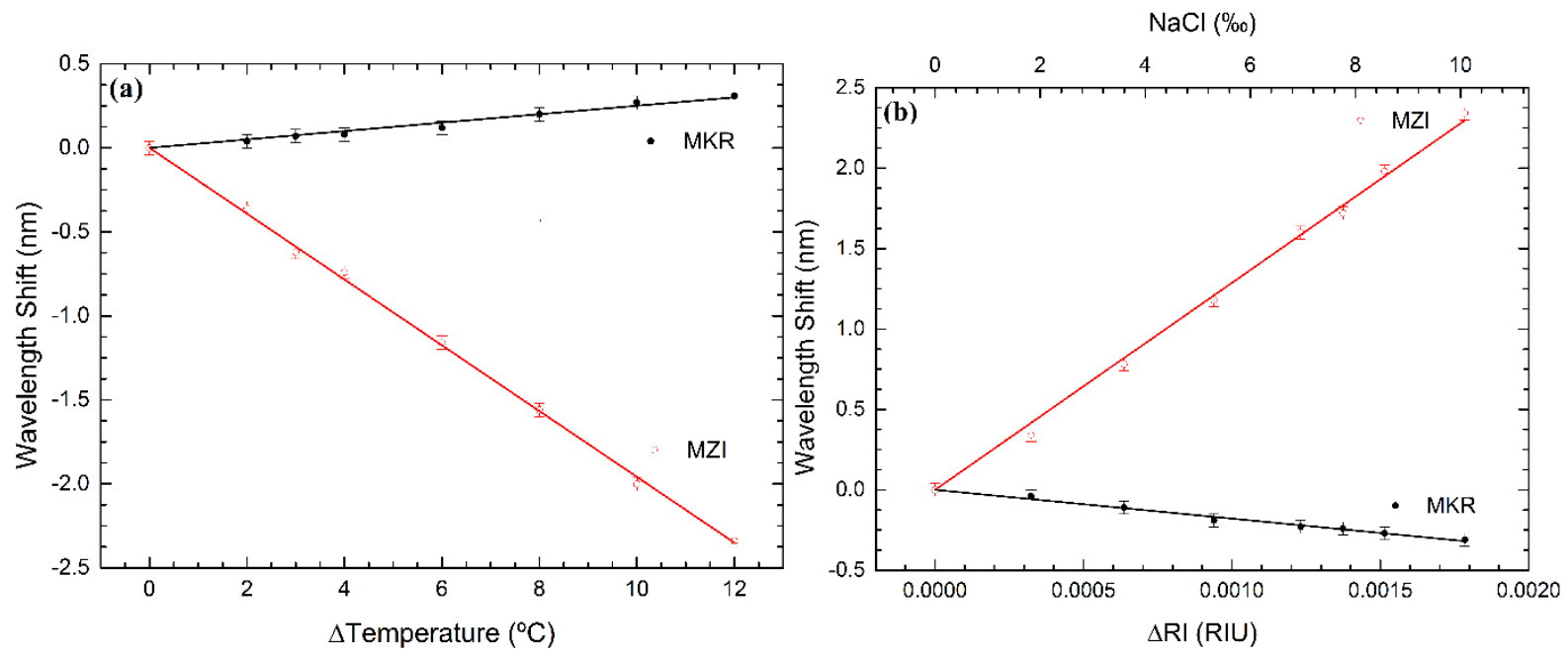

Figure 3. (a) Wavelength shift as a function of temperature variation for both components (MKR and MZI). (b)

Wavelength shift as a function of refractive index variation and $\mathrm{NaCl}$ concentration (per mil) for both components (MKR and MZI).

A simple matrix method using the sensitivities of both components (MZI and MKR) can be used to obtain simultaneously two of these parameters. For example, refractive index and temperature can be obtained using the matrix:

$$
\left[\begin{array}{c}
\Delta \mathrm{n} \\
\Delta T
\end{array}\right]=-0.3597\left[\begin{array}{cc}
0.0251 & 0.196 \\
179 & 1287
\end{array}\right]\left[\begin{array}{c}
\Delta \lambda_{M Z I} \\
\Delta \lambda_{M K R}
\end{array}\right]
$$

where $\Delta \lambda_{M Z I}$ and $\Delta \lambda_{M K R}$ are in nanometers, and the obtained refractive index and temperature variation are in refractive index units and degrees Celsius, respectively.

\section{DISCUSSION}

In summary, a new compact sensing structure using two distinct optical devices: a microfiber knot resonator integrated in an abrupt taper-based Mach-Zehnder interferometer, was produced using the same technology $-\mathrm{CO}_{2}$ laser processing. The sensor response is the combined response of both components, the MKR and the MZI. These two different spectral 
components respond differently to each measured parameter. A characterization in temperature, refractive index, and $\mathrm{NaCl}$ concentration was performed. The device can be used for discrimination of temperature and refractive index or $\mathrm{NaCl}$ concentration using the relationship matrix between the different measured parameters.

\section{ACKNOWLEDGMENTS}

This work was supported by Project NanoSTIMA - North Portugal Regional Operational Programme (NORTE-01-0145FEDER-000016). André Gomes is funded by FCT (PD/BI/129000/2017).

\section{REFERENCES}

[1] Jiang, X., Tong, L., Vienne, G., Guo, X., Tsao, A., Yang, Q.., Yang, D., "Demonstration of optical microfiber knot resonators," Appl. Phys. Lett. 88(22), 2004-2007 (2006).

[2] Zeng, X., Wu, Y., Hou, C., Bai, J.., Yang, G., "A temperature sensor based on optical microfiber knot resonator," Opt. Commun. 282(18), 3817-3819 (2009).

[3] Yang, H., Wang, S., Liao, X., Wang, J., Wang, X., Liao, Y.., Wang, J., "Temperature Sensing in Seawater Based on Microfiber Knot Resonator," Sensors 14(3), 18515-18525 (2014).

[4] Lim, K.-S. S., Aryanfar, I., Chong, W.-Y. Y., Cheong, Y.-K. K., Harun, S. W.., Ahmad, H., "Integrated microfibre device for refractive index and temperature sensing," Sensors (Basel). 12(9), 11782-11789 (2012).

[5] Li, X., Ding, H., "A Stable Evanescent Field-Based Microfiber Knot Resonator Refractive Index Sensor," IEEE Photonics Technol. Lett. 26(16), 1625-1628 (2014).

[6] Xu, Z., Sun, Q., Li, B., Luo, Y., Lu, W., Liu, D., Shum, P. P.., Zhan, L., "Highly sensitive refractive index sensor based on two cascaded microfiber knots with vernier effect," Opt. Express 23(5), 6662-6672 (2015).

[7] Schubert, T., Haase, N.., Kück, H., "Refractive-index measurements using an integrated Mach-Zehnder interferometer," Sensors Actuators A 60(100), 108-112 (1997).

[8] Kieu, K. Q., Mansuripur, M., "Biconical fiber taper sensors," IEEE Photonics Technol. Lett. 18(21), 2239-2241 (2006).

[9] Yang, J., Jiang, L., Wang, S., Li, B., Wang, M., Xiao, H., Lu, Y.., Tsai, H., "High sensitivity of taper-based MachZehnder interferometer embedded in a thinned optical fiber for refractive index sensing.," Appl. Opt. 50(28), 55035507 (2011).

[10] Gomes, A. D.., Frazão, O., "Mach - Zehnder Based on Large Knot Fiber Resonator for Refractive Index Measurement," IEEE Photonics Technol. Lett. 28(12), 1279-1281 (2016).

[11] Li, L., Xia, L., Xie, Z., Hao, L., Shuai, B.., Liu, D., "In-line fiber Mach-Zehnder interferometer for simultaneous measurement of refractive index and temperature based on thinned fiber," Sensors Actuators A Phys 180, 19-24 (2012).

[12] Lu, P., Men, L., Sooley, K.., Chen, Q., "Tapered fiber Mach-Zehnder interferometer for simultaneous measurement of refractive index and temperature," Appl. Phys. Lett. 94(13), 3-5 (2009).

[13] Cao, Y., Liu, H., Tong, Z., Yuan, S.., Su, J., "Simultaneous measurement of temperature and refractive index based on a Mach-Zehnder interferometer cascaded with a fiber Bragg grating," Opt. Commun. 342, 180-183 (2015).

[14] Luo, H., Sun, Q., Xu, Z., Jia, W., Liu, D.., Zhang, L., "Microfiber-Based Inline Mach-Zehnder Interferometer for Dual-Parameter Measurement," IEEE Photonics J. 7(2), 1-8 (2015).

[15] Liao, Y., Wang, J., Wang, S., Yang, H.., Wang, X., "Simultaneous Measurement of Seawater Temperature and Salinity Based on Microfiber MZ Interferometer With a Knot Resonator," J. Light. Technol. 34(1), 5378-5384 (2016).

[16] International Critical Tables of Numerical Data, Physics, Chemistry and Technology, McGraw-Hill Book Company, New York and London (1930).

[17] "CRC Handbook of Chemistry and Physics", 86th Edition, J. Am. Chem. Soc. 128(16), 5585 (2006). 